PROCEEDINGS OF THE AMERICAN MATHEMATICAL SOCIETY

Volume 124, Number 11, November 1996

\title{
ON THE SET OF TOPOLOGICALLY INVARIANT MEANS ON AN ALGEBRA OF CONVOLUTION OPERATORS ON $L^{p}(G)$
}

\author{
EDMOND E. GRANIRER
}

(Communicated by Dale E. Alspach)

\begin{abstract}
Let $G$ be a locally compact group, $A_{p}=A_{p}(G)$ the Banach algebra defined by Herz; thus $A_{2}(G)=A(G)$ is the Fourier algebra of $G$. Let $P M_{p}=A_{p}^{*}$ the dual, $J \subset A_{p}$ a closed ideal, with zero set $F=Z(J)$, and $\mathbb{P}=\left(A_{p} / J\right)^{*}$. We consider the set $T I M_{\mathbb{P}}(x) \subset \mathbb{P}^{*}$ of topologically invariant means on $\mathbb{P}$ at $x \in F$, where $F$ is "thin." We show that in certain cases card $T I M_{\mathbb{P}}(x) \geq 2^{c}$ and $T I M_{\mathbb{P}}(x)$ does not have the WRNP, i.e. is far from being weakly compact in $\mathbb{P}^{*}$. This implies the non-Arens regularity of the algebra $A_{p} / J$.
\end{abstract}

\section{INTRODUCTION}

Let $G$ be a locally compact group with unit $e$ and left Haar measure $d x$. Let $A_{p}(G)=A_{p}$, the Figa-Talamanca-Gaudry-Herz algebra of $G$ (see [Hz] or [Gr3], [Gr4]), thus $A_{2}(G)=A(G)$ is the Fourier algebra of $G$ as in [Ey1].

Let $P M_{p}=P M_{p}(G)=A_{p}^{*}, P M_{2}=P M_{2}(G)=A(G)^{*}$ their Banach space duals (thus algebras of left convolution operators on $L^{p}(G)$ ). $P M_{p}$ is an $A_{p}$ module via $(u \cdot \phi, v)=(\phi, u v)$ for $u, v \in A_{p}$.

If $\phi \in P M_{p}$ let supp $\phi \subset G$ denote its support (see sequel). If $\mathbb{P} \subset P M_{p}$ is a closed subspace, let $\sigma(\mathbb{P})=\left\{x \in G ; \lambda \delta_{x} \in \mathbb{P}\right\}$, where for any bounded Borel measure on $G$ let $\lambda \mu \in P M_{p}$ be given by $(\lambda \mu, v)=\int v d \mu$ for $v \in A_{p}$, and $\delta_{x}$ be the point mass at $x$. Denote $E_{\mathbb{P}}(x)=\operatorname{ncl}\{\phi \in \mathbb{P} ; x \notin \operatorname{supp} \phi\} \quad($ ncl $=$ norm closure) and $\mathbb{P}_{c}=\operatorname{ncl}\{\phi \in \mathbb{P} ; \operatorname{supp} \phi$ is compact $\}$.

If $x \in \sigma(\mathbb{P})$ let $T I M_{\mathbb{P}}(x)=\left\{\psi \in \mathbb{P}^{*} ; 1=\left(\psi, \lambda \delta_{x}\right)=\|\psi\|, \psi=0\right.$ on $\left.E_{\mathbb{P}}(x)\right\}$. Prop. 1 of [Gr3], p. 42 shows that this definition is consistent with [Gr3], p. 39.

Our main interest in this paper is in this set. Ching Chou has proved in [Ch2] that if $G$ is second countable nondiscrete and $\mathbb{P}=P M_{2}(G)$, then card $T I M_{\mathbb{P}}(e)=2^{c}$, where $c$ is the cardinality of $R$, the real line. This result has been definitively improved by Zhiguo $\mathrm{Hu}[\mathrm{Hu}]$ who found the exact cardinality of $T I M_{\mathbb{P}}(e)$ for an arbitrary $G$ and $\mathbb{P}=P M_{2}(G)$. The method of proof in both results is $C^{*}$ algebraic and does not apply if $p \neq 2$.

We have proved in [Gr2], Theorem 2.7 that for any $1<p<\infty$, if $G$ is second countable nondiscrete and $\mathbb{P}=P M_{p}(G)$, then card $T I M_{\mathbb{P}}(e)=2^{c}$ and $T I M_{\mathbb{P}}(e)$

Received by the editors March 13, 1995 and, in revised form, May 8, 1995.

1991 Mathematics Subject Classification. Primary 43A22, 42B15, 22D15; Secondary 42A45, 43A07, 44A35, 22D25. 
does not have the WRNP, see [Sa1] (which roughly means that $T I M_{\mathbb{P}}(e)$ is far from being a weakly compact set in $\left.\mathbb{P}^{*}\right)$.

Improving results in [Gr3] we have obtained in [Gr4] results about the set $T I M_{\mathbb{P}}(x)$ for the case that $\sigma(\mathbb{P})$ is "thin" in $G$. For these we need a few more notations. If $F, H$ are subsets of $G$, let $\operatorname{int}_{H} F$ be the interior of $F \cap H$ in $H$, thus $x \in \operatorname{int}_{H} F$ iff for some open $V$ in $G, x \in V \cap H \subset F \cap H$. The set $S \subset R$ (the real line) is symmetric if there are $t_{n}>0$ such that $t_{n}>\sum_{n+1}^{\infty} t_{i}$ for all $n$ and $S=\left\{\sum_{1}^{\infty} \varepsilon_{i} t_{i} ; \varepsilon_{i}=0,1\right\}$. If in addition $\sum_{1}^{\infty}\left(t_{i+1} / t_{i}\right)^{2}<\infty$, then $S$ is ultrathin symmetric, [GMc], p. 333. Among the results obtained in [Gr4] is the

Theorem. Let $\mathbb{P} \subset P M_{P}(G)$ be a $w^{*}$ closed $A_{p}$ submodule, $\sigma(\mathbb{P})=F, a, b \in G$. Assume that $F$ is metrisable.

(a) If $x \in \operatorname{int}_{a H b} F$ for some closed nondiscrete subgroup $H$, then card $T I M_{\mathbb{Q}}(x)$ $\geq 2^{c}$, where $\mathbb{Q}=\mathbb{P}$ or $\mathbb{P}_{c}$.

(b) If $p=2, G$ contains $R$ (or $T$ ) as a closed subgroup and $S \subset R$ is a symmetric set such that $x \in a S b \subset F$, then $\operatorname{card} \operatorname{TIM}_{\mathbb{Q}}(x) \geq 2^{c}$, where $\mathbb{Q}=\mathbb{P}_{c}$ or $\mathbb{P}$.

What happens if $F$ is not metrisable? In particular what happens if $G$ is nonmetrisable, $F=G=H$, thus $\mathbb{P}=P M_{p}(G)$ ? Does, in this case, $T I M_{\mathbb{P}}(e)$ contain at least two elements?

We answer this question, and much more, by improving the above results to nonmetrisable $F$, and showing that $\operatorname{TIM}_{\mathbb{P}}(x)$ does not have the WRNP, if $x$ is as above.

The method of proof requires an impressive result of I. Zelmanov [Ze] which guarantees an adequate supply of nondiscrete metrisable subgroups in any nondiscrete $G$, and the above mentioned results of [Gr4]. Many thanks are due to Wistar Comfort for pointing out [Ze] to us. We will prove the

Theorem. Let $\mathbb{P}$ be a $w^{*}$ closed $A_{p}$ submodule of $P M_{p}(G), \sigma(\mathbb{P})=F, a, b \in G$. Let $\mathbb{P}_{c} \subset \mathbb{Q} \subset P M_{p}(G)$ be any norm closed $A_{p}$ submodule.

(a) If $1<p<\infty, H$ is a nondiscrete closed subgroup and $x \in \operatorname{int}_{a H b} F$, then $\operatorname{card} T I M_{\mathbb{Q}}(x) \geq 2^{c}$ and $T I M_{\mathbb{Q}}(x)$ does not have the WRNP.

(b) If $p=2, G$ contains $R$ (or $T$ ) as a closed subgroup, $S \subset R$ (or $T$ ) is a symmetric set and $x \in a S b \subset F$, then $\operatorname{card} T I M_{\mathbb{Q}}(x) \geq 2^{c}$ and $T I M_{\mathbb{Q}}(x)$ does not have the WRNP.

In particular, if $F=H=G, 1<p<\infty$ (thus $\mathbb{P}=P M_{p}(G)$ ), we get that $\operatorname{card} T I M_{\mathbb{P}}(x) \geq 2^{c}$ and $T I M_{\mathbb{P}}(x)$ does not have the WRNP, for any nondiscrete $G$.

We also note that for any nondiscrete $G$ there exists $\mathbb{P} \subset P M_{p}(G)$ as in the above theorem such that $\operatorname{card} T I M_{\mathbb{P}}(x)=2^{c}$, hence the above estimate for $\operatorname{card} T I M_{\mathbb{P}}(x)$ cannot be improved.

Furthermore we show that if $J \subset A_{p}(G)$ is a closed ideal such that $Z(J)=F$ satisfies (a) or (b) of the above theorem, then the Banach algebra $A_{p} / J$ is not Arens regular.

Some more notation: If $\mathbb{P} \subset P M_{p}(G)$, let $W_{\mathbb{P}}(x)=C\left(\lambda \delta_{x}\right)+E_{\mathbb{P}}(x), x \in G$. If $\phi \in P M_{p}=A_{p}^{*}$, then supp $\phi$ is the set of $x \in G$ such that for any open $V \subset G$ with $x \in V$ there is some $v \in A_{p}$ such that $\operatorname{supp} v \subset V$ and $(\phi, v) \neq 0$, [Hz]. For the definition of WRNP, see [Sa1] or [Gr2], p. 156. These, as well as other notations can be found in any of [Gr3], [Gr4] or [Hz]. 


\section{THE MAIN RESULTS}

Proposition 1. Every nonmetrisable locally compact group $G$ contains an infinite compact abelian metrisable subgroup.

Proof. $G$ contains a compact normal infinite subgroup $N$ (see [HR] (8.7)). By E.I. Zelmanov's impressive result ([Ze], Theorem 2) $N$ contains an infinite abelian subgroup whose closure $A$ is a compact abelian infinite subgroup. Now by Rudin's Theorem 7 ([Ru3], p. 203) $A$ contains an infinite compact metrisable subgroup.

Corollary 2. Let $G$ be a nondiscrete locally compact group. Then $G$ contains a separable metric nondiscrete closed subgroup.

Proof. If $G$ is metrisable, let $x_{n} \in G$ be distinct and such that $x_{n} \rightarrow e$. Let $H$ be the closed subgroup generated by $\left\{x_{n}\right\}$.

Proposition 3. Let $\mathbb{P} \subset \mathbb{R}$ be norm closed $A_{p}(G)$ submodules of $P M_{p}(G)$ such that $a \in \sigma(\mathbb{P})$. Then any $\psi \in T I M_{\mathbb{P}}(a)$ has an extension $\psi_{0} \in T I M_{\mathbb{R}}(a)$.

Proof. Let $\psi_{1} \in \mathbb{R}^{*}$ be a norm preserving extension of $\psi$, by Hahn-Banach. Then $1=\left\|\psi_{1}\right\|=\|\psi\|=\left(\psi, \lambda \delta_{a}\right)=\left(\psi_{1}, \lambda \delta_{a}\right)$ since $\lambda \delta_{a} \in \mathbb{P} \subset \mathbb{R}$. Let now $S=S(a)=$ $\left\{u \in A_{p} ; 1=\|u\|=u(a)\right\}$. Then pointwise multiplication in $A_{p}$ renders $S(a)$ into an abelian semigroup as readily checked. Let $M \in \ell^{\infty}(S)^{*}$ be a translation invariant mean. Define $\psi_{0} \in \mathbb{R}^{*}$ by $\left(\psi_{0}, \phi\right)=M\left(\psi_{1}, u \cdot \phi\right)$, where $\left(\psi_{1}, u \cdot \phi\right)$ is considered as a bounded function on $S$ (i.e. in $\ell^{\infty}(S)$ ). It is now routine to check that $\left(\psi_{0}, v \cdot \phi\right)=\left(\psi_{0}, \phi\right)$ for all $v \in S(a)$ and $\phi \in \mathbb{R}$. Now, by [Gr3], Proposition 1 , $\psi_{0} \in T I M_{\mathbb{R}}(a)$ is the required extension.

Corollary 4. $\operatorname{card} T I M_{\mathbb{R}}(a) \geq \operatorname{card} T I M_{\mathbb{P}}(a)$. Furthermore, if $T I M_{\mathbb{P}}(a)$ fails to have the WRNP, then so does $\operatorname{TIM}_{\mathbb{R}}(a)$.

Proof. Let $i: \mathbb{P} \rightarrow \mathbb{R}$ be the canonical imbedding. If $T I M_{\mathbb{R}}(a)$ has the WRNP, then by E. Saab's Corollary 2 ([Sa1], p. 310), $i^{*} T I M_{\mathbb{R}}(a)=T I M_{\mathbb{P}}(a)$ also has the WRNP, which cannot be.

The following is folklore.

Proposition 5. Let $X, Y$ be Banach spaces and $W_{1} \subset W_{2} \subset X$ closed subspaces and $Y_{0} \subset Y$ a finite dimensional subspace. Let $q: Y \rightarrow Y / Y_{0}, p_{j}: X \rightarrow X / W_{j}$ be the canonical maps. (a) If $p_{2}$ is not weakly compact, then $p_{1}$ is not weakly compact; (b) If $t: X \rightarrow Y$ is a nonweakly compact operator, then $q t: X \rightarrow Y / Y_{0}$ is not weakly compact.

Proof. (a) Let $p_{3}: X / W_{1} \rightarrow X / W_{2}$ be the canonical map. If $p_{1}$ is weakly compact, then so is $p_{2}=p_{3} p_{1}$. (b) There is a closed subspace $Y_{1}$, such that $Y=Y_{0} \oplus Y_{1}$, thus $Y / Y_{0} \approx Y_{1}$. Thus $q[I-q]$ is the projection on $Y_{1}\left[Y_{0}\right]$ resp. and $I-q$ is weakly compact. If $q t$ is weakly compact, so is $t=q t+(I-q) t$ which cannot be.

Theorem 6. Let $G$ be any locally compact group and $\mathbb{P} \subset P M_{p}(G)$ a $w^{*}$ closed $A_{p}$ submodule with $F=\sigma(\mathbb{P})$. Assume that for some nondiscrete closed subgroup $H$ and $a, b \in G$, int $t_{a H b} F \neq \emptyset$. If $x \in \operatorname{int}_{a H b} F$, then $\operatorname{card} T I M_{\mathbb{Q}}(x) \geq 2^{c}$ and $T I M_{\mathbb{Q}}(x)$ does not have the WRNP, for any norm closed $A_{p}$ module $\mathbb{Q}$ such that $\mathbb{P}_{c} \subset \mathbb{Q} \subset P M_{p}(G)$. 
Remarks. (1) If $F$ is metrisable, then our Corollary 7 of [Gr4] implies part of the above theorem. The above is an improvement in that $F$ need not be metrisable. In particular one can take for an arbitrary nondiscrete locally compact group $G=$ $H=F$ and get that $\mathbb{P}=P M_{p}(G)$ satisfies card $T I M_{\mathbb{P}}(x) \geq 2^{c}$ and $T I M_{\mathbb{P}}(x)$ does not have the WRNP (a fortiori is not weakly compact in $P M_{p}(G)^{*}$ ) for any $x \in G$. If $p \neq 2$ this is a new result. The $C^{*}$ algebra methods of $\mathrm{Z}$. Hu in [Hu] do not seem to work in this case.

(2) One cannot improve the cardinality estimate of Theorem 6 . For assume that $1<p<\infty$, and $G$ is an arbitrary nondiscrete locally compact group. Then by Corollary $2 G$ contains a closed separable metrisable nondiscrete subgroup $H$. By Herz's theorem $[\mathrm{Hz}]$, p. 92 the restriction map $r: A_{p}(G) \rightarrow A_{p}(H)$ is onto and $\|r\| \leq 1$. Hence $r^{*} P M_{p}(H)=\mathbb{P}$ is norm closed and by Theorem 4.14 in [Ru1], $\mathbb{P}$ is $w^{*}$ closed. $\mathbb{P}$ is an $A_{p}$ module, since $\left(v \cdot r^{*} \phi, u\right)=\left(r^{*}[(r v) \cdot \phi], u\right)$ and $\sigma(\mathbb{P}) \subset H$, see $[\mathrm{Hz}]$. But $\sigma(\mathbb{P})=H$ since if $x \in H$ and $\phi=\lambda \delta_{x} \in P M_{p}(H)$, then $r^{*} \phi=\lambda \delta_{x} \in \mathbb{P}$. Now $A_{p}(H)$ is norm separable since $H$ is separable metric. Since $r^{*}$ is an isometry into (see [Hz], p. 91), card $P M_{p}(H)=$ card $\mathbb{P} \leq c$. Hence card $\mathbb{P}^{*} \leq 2^{c}$. We can apply now our Theorem 6 to $\mathbb{P} \subset P M_{p}(G), \sigma(\mathbb{P})=H=F$ and $a=b=e$ and get that $2^{c} \geq \operatorname{card} \mathbb{P}^{*} \geq \operatorname{card} T I M_{\mathbb{P}}(x) \geq 2^{c}$ for all $x \in H$. Thus card $T I M_{\mathbb{P}}(x)=2^{c}$.

Proof. Let $V \subset G$ be open such that $\operatorname{cl} V$ is compact and $x \in V \cap a H b \subset F$. Let, by Corollary $2, H_{0}$ be a nondiscrete separable closed metrisable subgroup of $G$. Then $x=a h b$ for some $h \in H$. Thus $x \in V \cap a h H_{0} b \subset V \cap a H b \subset F$. Let $F_{0}=$ $\operatorname{cl}\left(V \cap a h H_{0} b\right)$, a compact metrisable subset of $F$. If $c=a h$, then $x \in \operatorname{int}_{c H_{0} b} F_{0}$ since $x \in V \cap c H_{0} b \subset F_{0}$. Let $\mathbb{P}_{0}=w^{*} \operatorname{cllin}\left\{\lambda \delta_{x} ; x \in F_{0}\right\}, \mathbb{P}_{1}=w^{*} \operatorname{cllin}\left\{\lambda \delta_{x} ; x \in F\right\}$ where cl denotes closure and lin, linear span. Then $\mathbb{P}_{0} \subset\left(\mathbb{P}_{1}\right)_{c} \subset \mathbb{P}_{c}$, since $F_{0}$ is compact and since $\mathbb{P}_{1}$ is the smallest $w^{*}$ closed $A_{p}$ module with $\sigma\left(\mathbb{P}_{1}\right)=F,[\mathrm{~Hz}]$.

Apply now our Theorem 3 of [Gr4] to $\mathbb{P}_{0}$, the metrisable set $F_{0}$ and the closed nondiscrete group $H_{0}$. Then $\sigma\left(\mathbb{P}_{0}\right)=F_{0}, x \in \operatorname{int}_{c H_{0} b} F_{0}$, thus $x \in D_{1}\left(J_{0}\right)$ (see [Gr4]) where $J_{0}=\left\{u \in A_{p} ;(\phi, u)=0\right.$, for $\left.\phi \in \mathbb{P}_{0}\right\}$, a closed ideal such that $\mathbb{P}_{0}=\left(A_{p} / J_{0}\right)^{*}$. By Theorem 4 of $[\mathrm{Gr} 4]$ we get that there is some onto operator $t: \mathbb{P}_{0} \rightarrow \ell^{\infty}$ (thus $t^{*}$ is a $w^{*}-w^{*}$ and norm isomorphism into) such that $t^{*} \mathcal{F} \subset$ $T I M_{\mathbb{P}_{0}}(x)$ where $\mathcal{F}=\left\{\eta \in \ell^{\infty *} ; 1=(\eta, 1)=\|\eta\|\right.$ and $\eta=0$ on $\left.c_{0}\right)$.

Let now $\beta \mathbb{N}$ be the Stone-Cech compactification of the positive integers $\mathbb{N}$. Then $\beta \mathbb{N} \sim \mathbb{N} \subset \mathcal{F}$ is a $w^{*}$ perfect set of cardinality $2^{c}$ (see $[\mathrm{Ru} 3]$, p. 204). Thus $\Gamma=t^{*}(\beta \mathbb{N} \sim \mathbb{N})$ is a $w^{*}$ perfect subset of $T I M_{\mathbb{P}_{0}}(x)$ and card $T I M_{\mathbb{P}_{0}}(x) \geq 2^{c}$.

But $\Gamma$ is isomorphic to a canonical $\ell^{1}$ basis, i.e. there is some $d>0$ such that $\sum_{1}^{n}\left|\alpha_{i}\right| \geq\left\|\sum_{1}^{n} \alpha_{i} t^{*} \varphi_{i}\right\| \geq d \sum_{1}^{n}\left|\alpha_{i}\right|$ for all $n \geq 1, \alpha_{i} \in C$ and distinct $\varphi_{1}, \ldots, \varphi_{n} \in$ $\beta \mathbb{N} \sim \mathbb{N}$. To prove this it is enough (since $\left\|t^{*}\right\| \leq 1$ and $t^{*}$ is a norm isomorphism into) to show that $\sum_{1}^{n}\left|\alpha_{i}\right|=\left\|\sum_{1}^{n} \alpha_{i} \varphi_{i}\right\|$. Now $\ell^{\infty}=C(\beta \mathbb{N})$, hence there is some $f \in C(\beta \mathbb{N})$ such that $\|f\|=1$ and $f\left(\varphi_{i}\right)=\bar{\alpha}_{i} /\left|\alpha_{i}\right|$. Thus $\left(f, \sum_{1}^{n} \alpha_{i} \varphi_{i}\right)=\sum_{1}^{n}\left|\alpha_{i}\right| \leq$ $\left\|\sum_{1}^{n} \alpha_{i} \varphi_{i}\right\| \leq \sum_{1}^{n}\left|\alpha_{i}\right|$. It follows that the $w^{*}$ compact set $T I M_{\mathbb{P}_{0}}(x)$ contains a $w^{*}$ perfect set which is isomorphic to a canonical $\ell^{1}$ basis. Hence by our Lemma 1.2 on p. 157 in [Gr2], $T I M_{\mathbb{P}_{0}}(x)$ does not have the WRNP. To get the result about $T I M_{\mathbb{Q}}(x)$ apply Corollary 4.

With a view to future applications, we have under the assumptions of Theorem 6 :

Corollary 6'. Let $W \subset W_{\mathbb{Q}}(x)$ be any closed subspace. Then the canonical map $q: \mathbb{Q} \rightarrow \mathbb{Q} / W$ is not a weakly compact operator, for any $x \in \operatorname{int}_{a H b} F$. 
Proof. By Proposition $5\left(\right.$ a) we can assume that $W=W_{\mathbb{Q}}(x)$. Since $\mathbb{Q} / W_{\mathbb{Q}}(x)=$ $\left(\mathbb{Q} / E_{\mathbb{Q}}(x)\right) / C\left(\lambda \delta_{x}\right)$ and by Proposition $5(\mathrm{~b})$ we need only show that the canonical $\operatorname{map} q: \mathbb{Q} \rightarrow \mathbb{Q} / E_{\mathbb{Q}}(x)$ is not weakly compact. If $q$ is weakly compact, then so is $q^{*}:\left(\mathbb{Q} / E_{\mathbb{Q}}(x)\right)^{*} \rightarrow \mathbb{Q}^{*}$. But then $\left\{\psi \in \mathbb{Q}^{*} ;\|\psi\| \leq 1\right.$ and $\psi=0$ on $\left.E_{\mathbb{Q}}(x)\right\}$ and a fortiori $T I M_{\mathbb{Q}}(x)$ is a weakly relatively compact subset of $\mathbb{Q}^{*}$. But $T I M_{\mathbb{Q}}(x)$ is $w^{*}$, hence weakly, closed. Thus $T I M_{\mathbb{Q}}(x)$ is weakly compact and a fortiori has the WRNP. This cannot be by Theorem 6 .

We do not know if the next theorem holds for $p \neq 2$.

Theorem 7. Let $G$ be a locally compact group, and $\mathbb{P} \subset P M_{2}(G)$ a $w^{*}$ closed $A_{2}(G)$ module with $F=\sigma(\mathbb{P}), a, b \in G$. Assume that $R$ (or $T$ ) is a closed subgroup of $G$ and $S \subset R$ (or $T$ ) is a symmetric set such that $a S b \subset F$. If $x \in a S b$, then $\operatorname{card} T I M_{\mathbb{Q}}(x) \geq 2^{c}$ and $T I M_{\mathbb{Q}}(x)$ does not have the WRNP for any norm closed $A_{2}(G)$ module $\mathbb{Q}$ such that $\mathbb{P}_{c} \subset \mathbb{Q} \subset P M_{2}(G)$.

Remarks. If $F$ is metrisable, then Corollary 6 of [Gr4] implies the fact that $\operatorname{card} T I M_{\mathbb{Q}}(x) \geq 2^{c}$.

Proof. $S$ is a compact subset of $R$, since the map $t: \prod_{1}^{\infty} D_{i} \rightarrow S, D_{i}=\{0,1\}$, $t \varepsilon=\sum_{1}^{\infty} \varepsilon_{i} t_{i}$ is continuous. Let $F_{0}=a S b$, a compact metrisable subset of $a R b$, hence of $G$. Let $\mathbb{P}_{0}=w^{*}$ cl lin $\left\{\lambda \delta_{x} ; x \in F_{0}\right\}$. Then since $\sigma\left(\mathbb{P}_{0}\right)=F_{0}, \mathbb{P}_{0} \subset \mathbb{P}_{c}$. We can apply Corollary $2^{\prime}$ of [Gr4] with $F_{0}$ instead of $F$ and get that $x \in D_{1}\left(J_{0}\right)$, where $J_{0}=\left\{u \in A(G) ;(\phi, u)=0\right.$ for $\left.\phi \in \mathbb{P}_{0}\right\}$. Hence by [Gr4], Theorem 4, there is an onto operator $t: \mathbb{P}_{0} / W_{\mathbb{P}_{0}}(x) \rightarrow \ell^{\infty}$ such that $t^{*} \mathcal{F} \subset T I M_{\mathbb{P}_{0}}(x)$. The proof of Theorem 6 shows that card $T I M_{\mathbb{P}_{0}}(x) \geq 2^{c}$ and $T I M_{\mathbb{P}_{0}}(x)$ does not have the WRNP. Apply now Corollary 4 to $\mathbb{Q}$.

Remarks. (1) Theorem 7 holds true if $S=\bigcup_{\alpha \in I}\left(x_{\alpha}+S_{\alpha}\right)$, where $x_{\alpha} \in R, S_{\alpha}$ or $-S_{\alpha}$ are ultrathin symmetric and $I$ is any index set. Symmetric sets are such. This holds since Corolllary $2^{\prime}$ of [Gr4] holds for such sets $S$.

(2) If $F=G$ thus $\mathbb{P}=P M_{2}(G)$ and $x=e$, a much better and definitive result on $\operatorname{card} T I M_{\mathbb{P}}(e)$ has been obtained by Zhiguo $\mathrm{Hu}[\mathrm{Hu}]$.

(3) One cannot improve the cardinality estimate of Theorem 7. Indeed, $R$ (or $T$ ) is a closed subgroup of the otherwise arbitrary group $G$. Thus $R$ (or $T$ ) is a set of synthesis for $G$ (see $[\mathrm{Hz}])$. Thus $P M_{2}(R)\left(P M_{2}(T)\right)$ can be identified with $\mathbb{P}_{1}=\left\{\phi \in P M_{2}(G)\right.$; supp $\left.\phi \subset R\right\}$, a $w^{*}$ closed $A_{2}(G)$ submodule of $P M(G)$ with $F=\sigma\left(\mathbb{P}_{1}\right)=R($ or $T)$, see $[\mathrm{Hz}]$. Thus $\mathbb{P}_{1} \approx L^{\infty}(R)\left(\right.$ or $\left.\mathbb{P}_{1} \approx \ell^{\infty}\right)$. Now let $F=a S b$ and $\mathbb{P}=w^{*}$ cl lin $\left\{\lambda \delta_{x} ; x \in a S b\right\}$. Then $\mathbb{P} \subset \mathbb{P}_{1}$ and card $\mathbb{P} \leq \operatorname{card} \mathbb{P}_{1}=c$. But Theorem 7 implies $2^{c} \leq \operatorname{card} T I M_{\mathbb{P}}(x) \leq \operatorname{card} \mathbb{P}^{*} \leq \operatorname{card} \mathbb{P}_{1}^{*}=2^{c}$ for all $x \in a S b$.

Corollary $7^{\prime}$. With assumptions as in Theorem 7 let $W \subset W_{\mathbb{Q}}(x)$ be any closed subspace. Then the canonical map $q: \mathbb{Q} \rightarrow \mathbb{Q} / W$ is not a weakly compact operator, for any $x \in a S b$.

Proof. See the proof of Corollary $6^{\prime}$.

Corollary 8. Let $J \subset A_{p}(G)$ be a closed ideal, $F=Z(J)=\{x \in G ; v(x)=0$ for $v \in J\}, a, b \in G$. Assume one of the following:

(a) For some nondiscrete closed subgroup $H \subset G$, int $t_{a H b} F \neq \emptyset$ or

(b) $p=2, G$ contains $R$ (or $T$ ) as a closed subgroup, $S \subset R$ (or $T$ ) is a symmetric set such that $a S b \subset F$.

Then $A_{p} / J$ is a Banach algebra which is not Arens regular. 
Proof. Let $\mathbb{P}=(A / J)^{*}$. Then $W=W A P(\mathbb{P}) \subset W_{\mathbb{P}}(x)$ for $x \in \operatorname{int}_{a H b}(x \in a S b$ resp.), by [Gr4], Proposition 5 . But then $q: \mathbb{P} \rightarrow \mathbb{P} / W$ is not weakly compact, thus clearly $\mathbb{P} / W \neq\{0\}$. Hence $A / J$ is not Arens regular.

Remarks. (1) As shown by J.P. Kahane, there exist perfect sets $F \subset G=T$ (and even continuous curves $F$ (in $\operatorname{Lip} \beta, \beta<1$ ) in $G=R^{2}$ ) such that $A(G) / I_{F}=A(F)=$ $C(F)$, an Arens regular Banach algebra, where $I_{F}=\{v \in A(G) ; v=0$ on $F\}$. If $\mathbb{P}=A(F)^{*}$, one has in this case, card $T I M_{\mathbb{P}}(x)=1$ for all $x \in F$ (see [Gr3], p. $56-57)$.

(2) If $J$ satisfies the conditions of Corollary 8 and $G$ is second countable, then $A_{p} / J$ is even an extremely non-Arens regular (ENAR) Banach algebra, i.e. there is a closed subspace of $\mathbb{P} / W A P(\mathbb{P})$ which has $\mathbb{P}$ as a quotient. We do not know if this is the case if $G$ is not second countable.

\section{THE ABELIAN CASE}

If $G$ is abelian (l.c.a.), the above results have implications on translation invariant (tr. inv.) subspaces $P \subset L^{\infty}(\widehat{G})$. Denote $L^{\infty}(\widehat{G})=L^{\infty}, L^{1}(\widehat{G})=L^{1}, U C$ the uniformly continuous functions in $L^{\infty}, U C_{P}=U C \cap P, \bar{P}=\{\bar{f} ; f \in P\}$ and $\sigma(P)=\bar{P} \cap G$, where $G \subset L^{\infty}$ (are the continuous characters on $\widehat{G}$ ). Let $M_{P}(x)=$ $\left\{\psi \in P^{*} ; 1=\|\psi\|=(\psi, \bar{x})\right\}$. Let $\operatorname{TIM}_{P}(x)=\left\{\psi \in M_{P}(x) ;(\psi, f)=(\psi,(\bar{x} h) * f)\right.$ if $\left.f \in P, 0 \leq h \in L^{1}, \int h d \chi=1\right\}$ and $I M_{P}(x)=\left\{\psi \in M_{P}(x) ;(\psi, f)=x(\chi)\left(\psi, f_{\chi}\right)\right.$ for $\chi \in \widehat{G}, f \in P\}$, where $f_{\chi}(\gamma)=f(\chi \gamma)$. Recall that by Proposition 9 in [Gr4], $I M_{Q}(x) \supset T I M_{Q}(x)$ with equality if $Q \subset U C$.

Corollary 9. Let $G$ be l.c.a., $P[Q]$ be $w^{*}\left[\right.$ norm] closed tr. inv. subspaces of $L^{\infty}$ such that $U C_{P} \subset Q \subset L^{\infty}, F=\sigma(P), a \in G$. Assume that either

(a) for some nondiscrete closed subgroup $H \subset G$, int $t_{a H} F \neq \emptyset$ or

(b) $G$ contains $R$ (or $T$ ) as a closed subgroup, and $S \subset R$ (or $T$ ) is a symmetric set such that $a S \subset F$.

Then for any $x \in \operatorname{int}_{a H} F[x \in a S] \operatorname{card} I M_{Q}(x) \geq \operatorname{card} T I M_{Q}(x) \geq 2^{c}$ and both $I M_{Q}(x)$ and $T I M_{Q}(x)$ do not have the WRNP.

Proof. By [Sa1], p. 308 it is enough to prove the result for $T I M_{Q}(x)$. But by [Gr4] $\mathcal{F}^{* *}: L^{\infty *} \rightarrow P M(G)^{*}$ is a norm and $w^{*}-w^{*}$ linear homeomorphism such that $\mathcal{F}^{* *} T I M_{Q}(x)=T I M_{\mathbb{Q}}(x)$, where $\mathcal{F}^{*} \mathbb{Q}=Q$ and $\mathcal{F}: L^{1} \rightarrow A(G)$ is Fourier transform (see [Gr4]). Then as in [Gr4] and by the above Theorem 6, [7], card $T I M_{\mathbb{Q}} \geq 2^{c}$ and $T I M_{\mathbb{Q}}(x)$ does not have the WRNP. Hence again by [Sa1], p. 308 the same holds for $\operatorname{TIM}_{Q}(x)$.

Remarks. (1) The cardinality part of the above result is implied by Corollaries 10, 11 in [Gr4], in case $F$ is metrisable.

(2) Let $F \subset R=G, \chi_{t}(x)=e^{i t x}$ and $P_{*}(F)=w^{*}$ cllin $\left\{\chi_{t} ; t \in F\right\} \subset L^{\infty}(\widehat{G})$.

(a) Assume that $F$ is the Cantor $1 / 3$ set; thus for $t_{n}=2 / 3^{n}, F=\left\{\sum_{1}^{\infty} \varepsilon_{i} t_{i}\right.$; $\left.\varepsilon_{i}=0,1\right\}$, a symmetric set. Then for $t \in F$ and $P=P_{*}(F), T I M_{P}(t)=\left\{\psi \in P^{*}\right.$; $1=\|\psi\|=\psi\left(\bar{\chi}_{t}\right),\left(\psi, f_{x}\right)=\overline{\chi_{t}(x)}(\psi, f)$ for all $\left.f \in P, x \in R\right\}$, where $f_{x}(y)=$ $f(x+y)$, since $F$ is compact and by [Gr4], Proposition 9 . Then, since card $L^{\infty}(R)^{*}=$ $2^{c} \geq \operatorname{card} T I M_{P}(t)$, Corollary 9 yields that card $T I M_{P}(t)=2^{c}$ and $T I M_{P}(t)$ does not have the WRNP for any $t \in F$. Note that if $t=0$, then $T I M_{P}(0)=I M_{P}(0)$, the set of honest to goodness invariant means on $P$. 
(b) Assume that $F \subset R$ is a compact perfect Helson $S$ set with $0 \in F$. There exist such by $[\mathrm{He}]$ (or see [Ru1]). In this case, with $P=P_{*}(F)$ we have that $\operatorname{card} T I M_{P}(t)=1$, and $T I M_{P}(t)$ contains one element, hence certainly has the WRNP, for all $t \in F$. In particular for $t=0$, card $I M_{P}(0)=1$, for the set of honest to goodness invariant means on $P$ (see [Gr3]). The same is the case if $F \subset R$ is any scattered compact set, by Loomis' lemma [Lo].

Question: Does there exist a perfect set $F \subset R$ such that for $P=P_{*}(F)$, there is some $t_{0}$ in $F$ for which $\operatorname{card} \operatorname{TIM}_{P}\left(t_{0}\right)=c$ ?

\section{REFERENCES}

[BL] Y. Benyamini and P.K. Lin, Norm one multipliers on $L^{p}(G)$, Arkiv for Matematik 24 (1986), 159-173. MR 88e:42015

[Ch1] Ching Chou, Weakly almost periodic functions and Fourier Stiltjes algebras of locally compact groups, Trans. Amer. Math Soc. 274 (1982), 141-157. MR 84a:43008

[Ch2] - Topological invariant means on the Von Neumann algebra VN $(G)$, Trans. Amer. Math. Soc. 273 (1982), 207-229. MR 83k:20016

[Cow] M. Cowling, An application of Littlewood-Paley theory in Harmonic Analysis, Math. Ann. 241 (1979), 83-96. MR 81f: 43003

[DU] J. Diestel and J.J. Uhl Jr., Vector Measures, Amer. Math. Soc. Math Surveys No. 15, 1977. MR 56:12216

[Ey1] P. Eymard, L'algèbre de Fourier d'un groupe localement compact, Bull. Soc. Math. France 92 (1964), 181-236. MR 37:4208

[Ey2] P. Eymard, Algèbres $A_{p}$ et convoluteurs de $L^{p}$, Seminaire Bourbaki 22e année, 1969/70, no. 367, Lecture Notes in Math., vol. 180, Springer, New York, 1971. MR 42:7461

[FG] A. Figa-Talamanca and G.I. Gaudry, Density and representation theorems for multipliers of type $(p, q)$, J. Australian Math. Soc. 7 (1967), 1-6. MR 35:666

[GMc] C.C. Graham and O. Caruth McGehee, Essays in commutative harmonic analysis, Springer-Verlag, New York, 1979. MR 81d:43001

[Gr1] E.E. Granirer, On some spaces of linear functionals on the algebras $A_{p}(G)$ for locally compact groups, Colloq. Math. 52 (1987), 119-132. MR 88k:43006

[Gr2] Geometric and topological properties of certain $w^{*}$ compact convex subsets of double duals of Banach spaces which arise from the study of invariant means, Illinois J. Math. 30 (1986), 148-174. MR 87f:43001

[Gr3] On convolution operators with small support which are far from being convolution by a bounded measure, Colloq. Math. 67 (1994), 33-60. CMP 94:17

[Gr4] Day points for quotients of the Fourier algebra $A(G)$, extreme nonergodicity of their duals and extreme non Arens regularity, Illinois J. Math. (to appear).

[He] H. Helson, Fourier transforms on perfect sets, Studia Math. 14 (1954), 209-213. MR 16:817

[HR] E. Hewitt and K.A. Ross, Abstract Harmonic Analysis Vols. I,II, Springer-Verlag, 1963, 1970. MR 28:158; MR 41:7378

[Hu] Zhiguo $\mathrm{Hu}$, On the set of topologically invariant means on the Von Neumann algebra $V N(G)$, Illinois J. Math. (to appear).

[Hz] C. Herz, Harmonic synthesis for subgroups, Ann. Inst. Fourier Grenoble 37 (1973), 91123. MR 50:7556

[LP] Anthony To-Ming Lau and Alan L.T. Paterson, The exact cardinality of topologically invariant means on amenable locally compact groups, Proc. A.M.S. 98 (1986), 75-80. MR 88c: 43001

[Lo] L.H. Loomis, The spectral characterisation of a class of almost periodic functions, Annals of Math. 72 (1960), 362-368. MR 22:11255

[Me] Y. Meyer, Recent advances in spectral synthesis, Conference on Harmonic Analysis. College Park, Maryland. 1971 Springer Lecture Notes in Math., No. 266, pp. 239-253. MR 52:14863

[Mc] O. Caruth McGehee, Helson sets in $T^{n}$, Conference on Harmonic Analysis. College Park, Maryland. 1971. Springer Lecture Notes in Math., No. 266, pp. 229-237. MR 52:14862 
[Pa] A.L.T. Paterson, Amenability, Amer. Math. Soc. Mathematical Surveys and Monographs No. 29, 1988. MR 90e:43001

[Ru1] W. Rudin, Fourier Analysis on Groups, John Wiley and Sons, 1960. MR 27:2808

[Ru2] , Functional Analysis, McGraw-Hill Inc., 1973. MR 51:1315

[Ru3] Averages of continuous functions on compact spaces, Duke Math. J. 25 (1958), 197-204. MR 20:4774

[Sa1] E. Saab, Some characterizations of weak Radon-Nikodým sets, Proc. A.M.S. 86 (1982), 307-311. MR 84g:46030

[Ze] E.I. Zelmanov, On periodic compact groups, Israel J. Math. 77 (1992), 83-95. MR 94e:20055

Department of Mathematics, The University of British Columbia, Vancouver, British Columbia, Canada V6T 1Z2

E-mail address: granirer@math.ubc.ca 